\title{
REACHING TARGET LIPID LEVELS IN PATIENTS AT HIGH RISK OF CARDIOVASCULAR EVENT: THE EXPERIENCE OF A CANADIAN TERTIARY CARE LIPID CLINIC
}

\author{
Jennifer A. Klinke' ${ }^{1}$, Filip Málek², Min Gao ${ }^{3}$, Dan Holmes ${ }^{1}$, Jiri J. Frohlich ${ }^{1}$ \\ ${ }^{1}$ St. Paul's Hospital Lipid Clinic, Vancouver, Canada \\ ${ }^{2}$ Department of Internal Medicine I, Faculty Hospital Kralovské Vinohrady,3rd School of Medicine, Charles University, Prague, Czech Republic \\ ${ }^{3}$ St. Paul's Hospital, Centre for Health Evaluation and Outcome Sciences, Vancouver, Canada
}

\begin{abstract}
SUMMARY
Objectives: To determine the proportion of high risk patients followed at a tertiary care lipid clinic who met recommended lipid targets and to identify predictors of reaching goal lipid levels.

Research design and methods: A retrospective cohort study of 502 high risk patients followed between 1983 and 2003. Clinical and demographic data and fasting lipid profiles were extracted from each patient's first two clinic visits as well as the most recent visit.

Results: All patients in this study were at high risk of cardiovascular events due to dyslipidemia. At "Visit 1", only 55 (11.0\%) of patients were at target TC/HDL-C < 4.0, and 97 (19.3\%) of patients met target LDL-C $<2.5$ mmol//. At "Visit 3", 229 (45.8\%) patients reached TC/HDL-C target, and $216(43.2 \%)$ patients were at LDL-C target. The mean change in lipid values between Visit 1 and Visit 3 was significant $(p=0.0002)$ for LDL-C and $(p<0.0001)$ for TC/HDL-C. The use of statins, niacin, or salmon oil were all significantly associated with reaching TC/HDL-C target and LDL-C target, as well male gender, diabetes mellitus and peripheral vascular disease were also associated with reaching LDL-C target. Increasing age and lower body mass index were associated with reaching goal TC/HDL-C.

Conclusions: The mean absolute changes in lipid values were significant and median lipid levels approached target levels in patients followed at specialized clinic, however the majority of high risk patients are not meeting goal lipid levels.
\end{abstract}

Key words: cardiovascular risk, lipid target, statins, specialized clinic

Address for correspondence: F. Málek, Department of Internal Medicine I, Faculty Hospital Královské Vinohrady, 3rd School of Medicine, Charles University, Šrobárova Street 50, 100 34, Prague 10, Czech Republic. E-mail: malek@fnkv.cz

\section{INTRODUCTION}

The hydroxymethylglutaryl-coenzym A reductase inhibitors (statins) have been proven effective in the prevention of cardiovascular disease (1-4). The Scandinavian Simvastatin Survival Study study was the first to show reduction in hard endpoints, such as cardiovascular events and death, in patients with known coronary artery disease (CAD) treated with a statin (1). Statins have also been proven useful in high risk patients without markedly elevated cholesterol $(3,4)$. Meta-analysis of 38 primary and secondary prevention trials found that for every $10 \%$ reduction in serum cholesterol, coronary heart disease mortality was reduced by $15 \%$ and total mortality risk by $11 \%(5)$. The Canadian recommendations for the management of dyslipidemia (6) suggest specific lipid targets based on the risk of cardiovascular events. Patients with known atherosclerosis (CAD), peripheral vascular disease (PVD), or cerebrovascular disease (CVD) are at high risk of recurrent events.
Furthermore, diabetes mellitus is considered a CAD equivalent: those with diabetes have the same risk of myocardial infarction as nondiabetic patients with previous myocardial infarction (7). The most recent Canadian recommendations for the management of dyslipidemia suggest that all high risk patients receive aggressive lipid-lowering therapy to attain a target low-density lipoprotein cholesterol (LDL-C) $<2.5 \mathrm{mmol} / \mathrm{l}$ and total cholesterol to highdensity lipoprotein ratio (TC/HDL-C) $<4.0(6,7)$.

Despite the known benefits of lipid lowering therapy in patients at risk of cardiovascular events, only the minority of patients are meeting recommended LDL-C and TC/HDL-C targets. A recent review of 4,888 patients with dyslipidemia from 5 regions in the United States found that only 38\% were meeting LDL-C targets (9). Furthermore, only $18 \%$ of those with known coronary artery disease were reaching LDL-C target. These findings suggest that more aggressive treatment is required to achieve targets. In the present study, we sought to determine the proportion of high risk patients followed at a tertiary care lipid clinic

This study was supported by a grant from Merck/Schering. 
who met recommended lipid targets. We also sought to identify independent predictors of reaching goal lipid levels.

\section{METHODS}

Retrospective review of almost patients who were followed in the St. Paul's Hospital Healthy Heart Lipid Clinic in Vancouver, British Columbia was conducted. Five hundred and two patients at very high risk for cardiovascular events were identified and the corresponding charts were reviewed. At the time of data collection, the previous Canadian dyslipidemia recommendations(10), published in 2000, were in effect. Data was extracted from records on clinic visits between the years 1983 and 2003. Clinical and demographic data, medication use, adverse effects, and fasting lipid profiles were extracted from each patient's first and second visit to the Lipid Clinic, as well as from the most recent clinic visit (hereafter, referred to as "Visit 3"). Adverse reactions included myalgias, elevated transaminases, GI upset, or other, as documented in the physician progress notes of each chart.

The absolute and percent changes in lipid levels were calculated as the change from baseline (Visit 1) to the final visit level (Visit 3). The number of patients achieving target levels of LDL-C and TC/HDL-C was also determined. Chi-square test and GEE (generalized estimation equation) logistic regression analyses were used to examine, respectively, the univariate an independent association between clinical and demographic factors and successful attainment of lipid targets. Adjusted odds ratios and their $95 \%$ confidence intervals were then calculated.

\section{RESULTS}

Of the 502 high risk patients the majority of our cohort were males $(68 \%)$ with a mean age of 56 years (Table 1). Lipid clinic visits took place between January 1983 and mid 2003. Median TC/HDL-C and LDL-C levels decreased at each clinic visit. The median TC/HDL was 6.0, 4.8, and 4.2, and the median LDL-C was $4.1,3.0$, and $2.7 \mathrm{mmol} / 1$ on clinic visits 1,2 , and 3 , respectively. The mean change in lipid values between Visit 1 and Visit 3 was $1.73 \mathrm{mmol} / \mathrm{l}(95 \% \mathrm{CI}: 2.63$ to $-0.83 ; \mathrm{p}=0.0002)$ for LDL-C and 2.06 (95\% CI: 2.43 to $-1.69 ; \mathrm{p}<0.0001)$ for TC/HDL-C. Although

Table 1. Patient characteristics at Visit 1

\begin{tabular}{|l|c|}
\hline & Total cohort $(\mathbf{n}=\mathbf{5 0 2})$ \\
\hline Men & $342(68 \%)$ \\
\hline Age $(\text { years) })^{*}$ & $56.0 \pm 10.6$ \\
\hline BMl $\left(\mathrm{kg} / \mathrm{m}_{\iota}\right)^{*}$ & $27.5 \pm 4.1$ \\
\hline CAD & $318(63 \%)$ \\
\hline DM & $168(34 \%)$ \\
\hline PVD & $94(19 \%)$ \\
\hline CVD & $52(10 \%)$ \\
\hline No CAD/DM/PVD/CVD & $1(0.2 \%)$ \\
\hline
\end{tabular}

Data are in $(\%)$ or *mean \pm SD
Table 2. Mean difference from lipid target

\begin{tabular}{|l|c|c|c|}
\hline Target & Visit 1 & Visit 2 & Visit 3 \\
\hline LDL-C $<2.5 \mathrm{mmol} / /$ & $\begin{array}{c}1.7 \pm 1.7 \\
(67.7 \%)\end{array}$ & $\begin{array}{c}0.7 \pm 1.2 \\
(28.8 \%)\end{array}$ & $\begin{array}{c}0.4 \pm 1.1 \\
(16.7 \%)\end{array}$ \\
\hline TC/HDL-C $<4$ & $2.8 \pm 3.6$ & $\begin{array}{c}1.4 \pm 3.6 \\
(34.0 \%)\end{array}$ & $\begin{array}{c}0.7 \pm 3.3 \\
(17.7 \%)\end{array}$ \\
\hline TG $<2.0 \mathrm{mmol} / /^{*}$ & $\begin{array}{c}(69.6 \% 4.6 \\
(78.2 \%)\end{array}$ & $\begin{array}{c}0.9 \pm 3.6 \\
(43.5 \%)\end{array}$ & $\begin{array}{c}0.5 \pm 2.4 \\
(22.7 \%)\end{array}$ \\
\hline
\end{tabular}

Data are mean \pm SD (mean percent difference)

* 2000 Recommendations for the management and treatment of dyslipidemia

the percentage of patients achieving recommended lipid targets increased with each clinic visit, less than half of our cohort reached the targets by the final visit. Only 229 (46\%) patients achieved the recommended TC/HDL-C $<4.0$, and 216 (43\%) patients met target LDL-C $<2.5 \mathrm{mmol} / 1$ at Visit 3 . However, the mean difference from target lipid levels by Visit 3 was small: mean percent difference from target LDL-C was $16.7 \%$ and from target TC/HDL-C was $17.7 \%$ (Table 2).

GEE logistic regression models were used to determine predictive factors for meeting lipid targets, accounting for all 3 visits, and adjusted odds ratios and their $95 \%$ confidence intervals were calculated (Tables 3 and 4). Women were less likely to achieve LDL-C target compared to men (adjusted odds ratio $[\mathrm{AOR}]=0.7$, $95 \% \mathrm{CI}: 0.5-0.9, \mathrm{p}=0.0217)$. Both statin use $(\mathrm{AOR}=4.6,95 \%$ CI: $3.3-6.3, \mathrm{p}<0.0001)$ and a history of diabetes $(\mathrm{AOR}=2.8$, $95 \%$ CI: $2.0-3.8, \mathrm{p}<0.001)$ were significantly associated with meeting LDL-C targets. Other factors significantly associated with

Table 3. Adjusted odds ratio (AOR) for meeting LDL-C target

\begin{tabular}{|l|c|c|c|}
\hline \multirow{2}{*}{ Significant factors } & \multicolumn{2}{|c|}{ AOR } & \multirow{2}{*}{ p-value } \\
\cline { 2 - 3 } & Estimate & $95 \% \mathrm{Cl}$ & \\
\hline Female & 0.7 & $0.5-0.9$ & 0.0217 \\
\hline Statin use & 4.6 & $3.3-6.3$ & $<0.0001$ \\
\hline Niacin use & 1.5 & $1.0-2.3$ & 0.0462 \\
\hline Salmon oil use & 1.8 & $1.1-2.7$ & 0.0120 \\
\hline History of PVD & 1.7 & $1.2-2.5$ & 0.0065 \\
\hline History of DM & 2.8 & $2.0-3.8$ & $<0.0001$ \\
\hline
\end{tabular}

Table 4. Adjusted odds ratio (AOR) for meeting TC/HDL-C target

\begin{tabular}{|l|c|c|c|}
\hline \multirow{2}{*}{ Significant factors } & \multicolumn{2}{|c|}{ AOR } & \multirow{2}{*}{ p-value } \\
\cline { 2 - 3 } & Estimate & $95 \% \mathrm{Cl}$ & \\
\hline Female & 1.4 & $1.0-2.0$ & 0.0677 \\
\hline Age, per 5 years & 1.2 & $1.1-1.3$ & $<0.0001$ \\
\hline BMl, per 5 kg/m² & 0.7 & $0.6-0.9$ & 0.0053 \\
\hline Statin use & 7.4 & $4.8-11.2$ & $<0.0001$ \\
\hline Niacin use & 3.6 & $2.4-5.5$ & $<0.0001$ \\
\hline Salmon oil use & 2.0 & $1.1-3.4$ & 0.0147 \\
\hline
\end{tabular}

This study was approved by the Ethics Review Board of St. Paul's Hospital. 
reaching LDL-C targets were the use of niacin and salmon oil, and a history of PVD. Older patients were more likely to achieve TC/HDL-C targets (AOR $=1.2$ for age increase of 5 years, $95 \% \mathrm{CI}$ : $1.1-1.3, \mathrm{p}<0.0001)$. Conversely, patients with an elevated BMI were less likely to achieve TC/HDL-C targets (AOR =" 0.7 for BMI increase of $\left.5 \mathrm{~kg} / \mathrm{m}_{\iota}, 95 \% \mathrm{CI}: 0.6-0.9, \mathrm{p}=0.0053\right)$. The use of statins, niacin, or salmon oil were all significantly associated with reaching TC/HDL targets $(\mathrm{AOR}=7.4,95 \% \mathrm{CI}$ : $4.8-11.2$, $\mathrm{p}<0.0001 ; \mathrm{AOR}=3.6,95 \% \mathrm{CI}: 2.4-5.5, \mathrm{p}<0.0001$; and AOR $=2.0,95 \%$ CI: $1.1-3.4, \mathrm{p}=0.0147$, respectively).

Over the course of follow-up, 35 (7.0\%) of patients experienced an adverse reaction: 25 patients had myalgias, 3 patients had elevated liver enzymes, 5 patients had GI upset, 2 patients reported fatigue, and 2 patients experienced other adverse effects.

\section{DISCUSSION}

All patients in the current study were considered to be at high risk of cardiovascular events. The Canadian recommendations for the management of dyslipidemia suggest targets of TC/HDL-C $<4.0$ and LDL-C $<2.5 \mathrm{mmol} / \mathrm{l}$ for these patients (6). Despite at least 3 visits to a specialized lipid clinic, we found that only $46 \%$ and $43 \%$ of patients were ultimately reaching target TC/HDL-C and LDL-C, respectively. On their most recent clinic visit, 90\% of patients were taking a statin drug and $36 \%$ were on combination therapy with other lipid-lowering agents. Furthermore, we found low utilization rates of other cardioprotective medications. At Visit 3, only $72.8 \%, 46.8 \%$, and $33.0 \%$ of these high risk patients were taking aspirin, ACE inhibitor, or beta blocker therapy, respectively. This latter finding suggests that patients (and perhaps physicians) may not always be compliant with recommended medical therapy. These findings are very similar to those found in a study of 136 patients carried out by cardiovascular risk reduction clinic in Edmonton (11) where the charts of all patients treated with combination therapy (statin-fibrate, or statin-niacin) were reviewed. In that study, after an average follow-up period of 18.5 months, only $43 \%$ of patients were achieving target levels for LDL-C. Most of these patients $(84.6 \%)$ were considered to be at high risk of cardiovascular events.

In another Czech overview of the status of cardiovascular pharmacotherapy in 857 patients after myocardial infarctions and/or myocardial revascularization in the Czech republic in 2005 compared to years 1999-2000 the proportion of patients treated with lipid-lowering therapy rose from $47.8 \%$ to $88.1 \%$ and the LDL cholesterol target $<2.5 \mathrm{mmol} / \mathrm{l}$ was achieved in $52.8 \%$ of patients with another $23.7 \%$ subjects with LDL cholesterol 2.5-3.0 mmol/1 (22).

Although less than half of our cohort had reached lipid targets at Visit 3, the median TC/HDL-C was 4.2 with a mean difference from target of $0.7 \pm 3.3(17.7 \%)$ and the median LDL-C was $2.7 \mathrm{mmol} / \mathrm{l}$ with a mean difference from target of $0.4 \pm$ $1.1 \mathrm{mmol} / 1(16.7 \%)$. Therefore, the majority of these patients were near target.

Statins, niacin, and salmon oil were all independently associated with reaching lipid targets. We found that patients who were taking a statin (alone or in combination with another lipid lowering agent) were more likely to be meeting lipid targets than patients not taking statins. Women were less likely to reach target
LDL-C than men but there was no significant gender difference for $\mathrm{TC} / \mathrm{HDL}-\mathrm{C}$. These findings may be due to the perception that men have a greater cardiovascular risk and are, therefore, treated more aggressively. The lack of difference for TC/HDL-C is likely because women tend to have a higher HDL-C than men. Interestingly, patients with diabetes or PVD (but not CAD or CVD) were more likely to reach target LDL-C. Diabetic patients tend to have one or more other specialists involved in their care and also may receive more frequent dietary counseling (outside the Lipid Clinic) than non-diabetic patients, which may contribute to the differences seen in this cohort. It is unclear why patients with PVD, but not CAD or CVD, would have more success at reaching LDL-C target. Increasing age was associated with reaching TC/HDL-C targets, and increasing body mass index (BMI) was negatively associated with reaching TC/HDL-C targets. Older patients may be more compliant, perhaps due to the perception of higher likelihood of suffering a cardiovascular event than younger patients.

There are several possible reasons to explain the finding that less than half of our cohort met lipid targets. First, there may have been inadequate dose titrations. Almost forty percent (39.8\%) of patients had an uptitration of a statin and $52.4 \%$ of patients were switched to a different statin during the time frame of this study. However, these values likely underestimate true rates of uptitration due to the design of our study. Second, the majority of our cohort was receiving monotherapy. Third, side effects may have precluded optimization of drug dosing. Although only $7.0 \%$ of our cohort experienced an adverse reaction, this may also be an underestimate given by our study design. And finally, patient non-compliance may have played a role; however, compliance was not specifically addressed in our retrospective study.

It is well known that statins lower LDL-cholesterol and TC/ HDL-C, promote regression of atherosclerotic lesions, and reduce cardiovascular events and mortality $(1-4,12,13)$. Often, however, treatment of dyslipidemia with monotherapy is ineffective in attaining goal lipid levels due to inadequate drug potency or drug titration, or drug intolerance at higher doses. The ACCESS trial was a randomized controlled trial of more than 3,000 patients with CAD (14).

Patients were randomized to receive one of five different statins, and doses were titrated at 6,12 , and 18 weeks (up to maximum doses) if target LDL-C was not met. After 54 weeks of monotherapy, many patients were not at target despite adequate titration: $72 \%$ of patients receiving atorvastatin were at LDL-C target, compared to $52 \%$ in the simvastatin group, $44 \%$ in the lovastatin group, $30 \%$ in the fluvastatin group, and $25 \%$ of the pravastatin group. Furthermore, one study examined high risk patients seen at a Veteran Affairs medical center and found that combination lipid-lowering therapy, rather than monotherapy, predicted achievement of goal lipid levels (15).

Numerous studies have demonstrated long-term efficacy and safety with combination lipid-lowering therapy (16-21). The HDL-Atherosclerosis Treatment Study (HATS) showed that a striking reduction in outcomes can be achieved by combination treatment (20). The combination of simvastatin and niacin resulted in a net regression of coronary atherosclerosis (assessed by quantitative angiography) and a 90\% reduction in cardiovascular events. Similarly, the Familial Atherosclerosis Treatment Study (FATS) demonstrated that lovastatin plus colestipol (a bile acid binding 
resin) and niacin plus colestipol led to regression of atherosclerosis and a $73 \%$ reduction in cardiovascular events (21).

Our study has two main limitations. First, it was a retrospective chart review, and may not be representative of patients followed in the community by other specialists or family doctors. Second, not all clinic visits were accounted for (i.e. some patients may have had several clinic visits between Visit 2 and the most recent visit [Visit 3]). Thus, not all drug regimens, dosing changes, or adverse effects were evaluated. However, the main purpose of this study was to identify the proportion of high risk patients who successfully met recommended lipid targets, and the absolute change in lipid levels between the initial and final clinic visits.

The majority of high risk patients, who benefit the most from intensive lipid-lowering, are currently not meeting recommended lipid targets. Both patients in the community and those attending specialized lipid clinics are not reaching targets, as documented in the current study and others $(9,11)$. Current recommendations for the management of dyslipidemias and secondary prevention of coronary artery disease in adults suggest new target for LDL-C $<2.0 \mathrm{mmol} / \mathrm{l}$ in high risk patients, e.g. subjects with established CAD and DM $(23,24)$. Using this new target, the proportion of patients not achieving recommended goal in our study would be much more higher.

More aggressive lipid-lowering is needed. Similar to the experience with drug treatment of hypertension, a combination of two or more agents may be required in a significant proportion of these patients. Options for combination therapy include statins, fibrates, bile acid sequestrants, niacin, or the newest lipid-lowering agent ezetimibe. Physicians, whether in the community or specialized clinics, must strive for more intensive lipid-lowering in order to reduce the morbidity and mortality in high risk patients.

\section{REFERENCES}

1. Pedersen TR, Kjekshus J, Berg K, Haghfelt T, Faergeman O, Faergeman G, et al; Scandinavian Simvastatin Survival Study Group. Randomised trial of cholesterol lowering in 4444 patients with coronary heart disease: the Scandinavian Simvastatin Survival Study (4S). Lancet. 1994;344(8934):1383-9.

2. Prevention of cardiovascular events and death with pravastatin in patients with coronary heart disease and a broad range of initial cholesterol levels. The Long-Term Intervention with Pravastatin in Ischaemic Disease (LIPID) Study Group. N Engl J Med. 1998 Nov 5;339(19):1349-57.

3. Sacks FM, Pfeffer MA, Moye LA, Rouleau JL, Rutherford JD, Cole TG, et al. The effect of pravastatin on coronary events after myocardial infarction in patients with average cholesterol levels. Cholesterol and Recurrent Events Trial investigators. N Engl J Med. 1996 Oct 3;335(14):1001-9.

4. Heart Protection Study Collaborative Group. MRC/BHF Heart Protection Study of cholesterol lowering with simvastatin in 20,536 high-risk individuals: a randomised placebo-controlled trial. Lancet. 2002 Jul 6;360(9326):7-22.

5. Gould AL, Rossouw JE, Santanello NC, Heyse JF, Furberg CD. Cholesterol reduction yields clinical benefit: impact of statin trials. Circulation. 1998 Mar 17;97(10):946-52.

6. Genest J, Frohlich J, Fodor G, McPherson R; Working Group on Hypercholesterolemia and Other Dyslipidemias. Recommendations for the management of dyslipidemia and the prevention of cardiovascular disease: summary of the 2003 update. CMAJ. 2003 Oct 28;169(9):921-4. Erratum in: CMAJ. 2003 Nov 25;169(11):1149.

7. Haffner SM, Lehto S, Ronnemaa T, Pyorala K, Laakso M. Mortality from coronary heart disease in subjects with type 2 diabetes and in nondiabetic subjects with and without prior myocardial infarction. N Engl J Med. 1998 Jul 23;339(4):229-34.

8. Expert Panel on Detection, Evaluation, and Treatment of High Blood Cholesterol in Adults. Executive Summary of The Third Report of The
National Cholesterol Education Program (NCEP) Expert Panel on Detection, Evaluation, and Treatment of High Blood Cholesterol in Adults (Adult Treatment Panel III). JAMA. 2001 May 16;285(19):2486-97.

9. Pearson TA, Laurora I, Chu H, Kafonek S. The lipid treatment assessment project (L-TAP): a multicenter survey to evaluate the percentages of dyslipidemic patients receiving lipid-lowering therapy and achieving low-density lipoprotein cholesterol goals. Arch Intern Med. 2000 Feb 28;160(4):459-67.

10. Fodor JG, Frohlich JJ, Genest JJ Jr, McPherson PR. Recommendations for the management and treatment of dyslipidemia. Report of the Working Group on Hypercholesterolemia and Other Dyslipidemias. CMAJ. 2000 May 16;162(10):1441-7.

11. Taher TH, Dzavik V, Reteff EM, Pearson GJ, Woloschuk BL, Francis GA. Tolerability of statin-fibrate and statin-niacin combination therapy in dyslipidemic patients at high risk for cardiovascular events. Am J Cardiol. 2002 Feb 15;89(4):390-4.

12. Downs JR, Clearfield M, Weis S, Withney E, Shapiro DR, Beere PA, et al. Primary prevention of acute coronary events with lovastatin in men and women with average cholesterol levels: results of AFCAPS/TexCAPS. Air Force/Texas Coronary Atherosclerosis Prevention Study. JAMA. 1998 May 27;279(20):1615-22.

13. Shepherd J, Cobbe SM, Ford I, Isles CG, Lorimer AR, MacFarlane PW, et al. Prevention of coronary heart disease with pravastatin in men with hypercholesterolemia. West of Scotland Coronary Prevention Study Group. N Engl J Med. 1995 Nov 16;333(20):1301-7.

14. Andrews TC, Ballantyne CM, Hsia JA, Kramer JH. Achieving and maintaining National Cholesterol Education Program low-density lipoprotein cholesterol goals with five statins. Am J Med. 2001 Aug 15;111(3):18591.

15. Schectman G, Hiatt J. Drug therapy for hypercholesterolemia in patients with cardiovascular disease: factors limiting achievement of lipid goals. Am J Med. 1996 Feb;100(2):197-204.

16. Athyros VG, Papageorgiou AA, Hatzikonstandinou HA, Didangelos TP, Carina MV, Kranitsas DF, et al. Safety and efficacy of long-term statin-fibrate combinations in patients with refractory familial combined hyperlipidemia. Am J Cardiol. 1997 Sep 1;80(5):608-13.

17. Ellen RL, McPherson R. Long-term efficacy and safety of fenofibrate and a statin in the treatment of combined hyperlipidemia. Am J Cardiol. 1998 Feb 26;81(4A):60B-65B.

18. Pauciullo P, Borgnino C, Paoletti R, Mariani M, Mancini M. Efficacy and safety of a combination of fluvastatin and bezafibrate in patients with mixed hyperlipidemia (FACT study). Atherosclerosis. 2001 Jun;150(2):429-36.

19. Blankenhorn DH, Nessim SA Johnson RL, Sanmarco ME, Azen SP, Cashin-Hemphill L. Beneficial effects of combined colestipol-niacin therapy on coronary atherosclerosis and coronary venous bypass grafts. JAMA. 1987 Jun 19;257(23):3233-40. Erratum in: JAMA. 1988 May 13;259(18):2698.

20. Brown BG, Zhao XQ, Chait A, Fisher LD, Cheung MC, Morse JS, et al. Simvastatin and niacin, antioxidant vitamins, or the combination for the prevention of coronary disease. N Engl J Med. $2001 \mathrm{Nov}$ 29;345(22):1583-92.

21. Brown G, Albers JJ, Fisher LD, Schaefer SM, Lin JT, Kaplan C, et al. Regression of coronary artery disease as a result of intensive lipid-lowering therapy in men with high levels of apolipoprotein B. N Engl J Med. 1990 Nov 8;323(19):1289-98.

22. Widimský J, Vaněk P, Juráň F, Lánská V. Current status of cardiovascular pharmacotherapy in patients after myocardial infarction and/or revascularization in the Czech Republic in 2005. Developments seen during the past 5 and 10 years. Cor Vasa. 2006;48(4):135-41.

23. Grundy SM, Cleeman JI, Merz CN, Brewer HB Jr, Clark LT, Hunninghake DB, et al; National Heart, Lung, and Blood Institute; American College of Cardiology Foundation; American Heart Association. Implication of recent clinical trials for the National Cholesterol Education Program Adult Treatment Panel III guidelines. Circulation. 2004 Jul 13,110(2):227-39. Erratum in: Circulation. 2004. Aug 10;110(6):763.

24. Cannon CP, Steinberg BA, Murphy SA, Mega JL, Braunwald E. Metaanalysis of cardiovascular outcomes trials comparing intensive versus moderate statin therapy. J Am Coll Cardiol. 2006 Aug 1;48(3):438-45. 